$(\mathrm{RII}=0.39-0.58)$. For females, SEI begins at $20-24$ years $(\mathrm{RII}=0.27)$ with pronounced inequalities at 60-79years and peaking at 65-69 years $(\mathrm{RII}=0.30-0.48)$. All four morphology groups demonstrate inequalities for lung cancer. For cervical cancer, squamous cell carcinoma dominates; in oesophageal cancer, squamous cell carcinoma followed by adenocarcinoma and ultimately other morphologies show inequalities. For head and neck cancers; hypopharynx, piriform sinus and larynx followed by lip, oral cavity and ultimately oropharynx, base of tongue, palate and tonsil show inequalities. We conclude: age, morphology, sex and site provide important information to better understand SEI.

\section{P1-330 INEQUALITIES IN DENTAL CARIES: THE ROLE OF NMES INTAKE}

doi:10.1136/jech.2011.142976f.22

\begin{abstract}
${ }^{1}$ A Sherriff, ${ }^{*} \mathrm{M}$ Wilson, ${ }^{2} \mathrm{~J}$ Armstrong, ${ }^{2} \mathrm{Y}$ Brogan, ${ }^{1} \mathrm{~L}$ Macpherson. ${ }^{1}$ Glasgow
\end{abstract} University, Glasgow, UK; ${ }^{2}$ Glasgow Caledonian University, Glasgow, UK

Introduction Dental caries is one of the most prevalent diseases of childhood in the UK, with a disproportionate burden experienced by the most disadvantaged groups. Non-milk extrinsic sugar (NMES) intake, poor oral hygiene and acidogenic bacteria are considered the main risk factors for caries, however, their role in explaining the observed inequalities has not been fully explored. The aim of the study is to assess the extent to which these factors explain the socio-economic (SES) inequalities in caries.

Methods Data on treatment for decay (caries), SES (Scottish Index of Multiple Deprivation (SIMD)), NMES intake and oral hygiene was obtained from 1491 children participating in the Survey of Sugar Intake among Children in Scotland (3-17 years). Logistic regression models assessed the impact on the Caries-SES relationship of NMES intake and oral hygiene. A priori interactions tests were performed.

Results $54 \%$ of children had caries and there was a strong SES gradient $(p<0.001)$. The OR $[95 \% \mathrm{CI}]$ for caries in the most deprived vs least deprived groups was 3.9 [2.8 to 5.5], and increased slightly when adjustments were made for NMES intake and oral hygiene (AOR $[95 \% \mathrm{CI}]=4.3$ [2.9 to 6.3]). There was no evidence of an interaction between NMES intake/Oral hygiene, SES and caries $(\mathrm{p}=0.4 ; \mathrm{p}=0.7)$.

Conclusions The SES patterning of caries is not attenuated by NMES intake and/or oral hygiene. Further work is required to explore alternative pathways to explaining the observed inequalities in oral health and may focus on the interaction between diet and acidogenic bacteria

\section{P1-331 OUTCOMES OF SCREENING MAMMOGRAPHIES IN ONE COMMUNITY-BASED INITIATIVE IN LEBANON}

doi:10.1136/jech.2011.142976f.23

J Sidaoui, ${ }^{*}$ S Adib. University of St Joseph, Beirut, Lebanon

Introduction and objectives There is no formal breast screening program currently in Lebanon. This report describes the outcomes of a screening mammography initiative implemented by one Lebanese non-governmental organization. The objectives were to assess the socio-demographic and reproductive characteristics associated with repeating a screening mammography after receiving reassuring results from a first one, and to estimate, for the first time in Lebanon, the proportion of mammographies suggesting malignancy which could be obtained from a mass screening activity.
Methods A total of 1500 women participated in the "Faire Face" screening initiative between 2002 and 2009, which allowed them to obtain repeated annual mammographies free of charge.

Results Of participants, 58\% repeated the test at least once even after receiving reassuring results. Factors associated with test repetition were older age, fewer children and retirement but not housewife or employed status. Results were suspicious (ACR4 or 5) in $2.4 \%$ of cases

Discussion and conclusions The proportion of suspicious readings on mammograms which require further investigation was similar to European figures. Evidence shows that, given the opportunity, increasingly more women of younger ages are willing to undergo the required annual test, and to repeat it. Several issues have to be debated nationally including lowering the financial barriers to mammography for all women regardless of their socio-demographic backgrounds, and improving the opening hours of mammography test centers to attract working women or those with larger families, and therefore with limited free time in the morning.

\section{P1-332 FIRST INSIGHT INTO GENETIC DIVERSITY OF MYCOBACTERIUM TUBERCULOSIS STRAINS IN SALVADOR, BRAZIL}

doi:10.1136/jech.2011.142976f.24

${ }^{1}$ A Silva, ${ }^{*}$ L Ferrazoli, ${ }^{1} \mathrm{~J}$ Reis, ${ }^{2} \mathrm{~S}$ Pereira, ${ }^{2} \mathrm{E}$ Mota, ${ }^{1} \mathrm{M}$ Reis. ${ }^{1}$ Centro de pesquisa gonçalo muniz, Fundação oswaldo cruz-bahia, Salvador, Bahia, Brazil; ${ }^{2}$ Universidade federal da Bahia, Instituto de saúde coletiva, Salvador, Bahia, Brazil; ${ }^{3}$ Instituto adolfo lutz, IAL, São Paulo, São Paulo, Brazil

This study constitutes a first attempt to describe the genetic population structure of $M$ tuberculosis circulating in Salvador. A total of 56 confirmed cases of pulmonary tuberculosis, identified between March and June, 2008 were analysed using IS6110-restriction fragment length polymorphism (RFLP). The study population was characterised by male (71.4\%) and over 30 years of age $(68.7 \%)$. Forty-one isolates were found in a single pattern (73.21\%), while 15 $(26.78 \%)$ were found in group patterns, forming six clusters. The higher rate of diversity observed is much more suggestive of endogenous reactivation than recent transmission.

\section{P1-333 YEARS OF POTENTIAL LIFE LOST FROM ASSAULT WITH FIREARMS OR SHARP OBJECTS AMONG ADOLESCENTS IN CAMPO GRANDE, MS, BRAZIL, IN 2007}

doi:10.1136/jech.2011.142976f.25

A P Silva, ${ }^{*}$ E R Pontes, J R Tognini. Universidade Federal de Mato Grosso do Sul, Campo Grande, Brazil

Introduction In Brazil, mortality by external causes has reached epidemic proportions, having become the principal cause of death among male adolescents (Brazil, 2010). The aim of this study was to evaluate the years of potential life lost by this adolescent group in Campo Grande, MS, Brazil, in 2007.

Methods This retrospective study investigated deaths caused by assault with firearm discharge or sharp objects, as described in Chapter XX of the ICD-10 (WHO, 1996). The proportion of these deaths among adolescents aged 15 to 19 years was calculated. Data were collected from the Datasus database of the Brazilian Unified Health Care System.

Results For every 1000 deaths occurring in this age range in 2007, 528 were caused by assault with firearms or sharp objects. Considering that life expectancy in Mato Grosso do Sul State is 73.8 years (IBGE, 2007), 58.8 years of potential life are lost by every 15 -year-old victim. 
Conclusion Such a high rate of premature mortality not only reflects social vulnerability, but also reveals the failure of intersectoral policies to control a situation that affects the prospects of adolescents, their families, and society as a whole. Epidemiological knowledge must translate to ethically oriented approaches by healthcare managers and governmental policymakers in order to minimise these deaths, which represent a serious health hazard and social disease in Brazil.

\section{P1-334 USE OF WEIGHT-LOSS DRUGS IN SOUTHERN BRAZIL: A POPULATION BASED STUDY}

doi:10.1136/jech.2011.142976f.26

E Machado, M Silveira, V M Silveira.* Faculty of Medicine, Federal University of Pelotas, Pelotas, Rio Grande do Sul, Brazil

Introduction Increasing prevalence of obesity worldwide reinforces the importance of studying weight-loss habits of the population. It is known that lifestyle modifications (LSM) should be the mainstay of overweight treatment and that use of drugs should be considered adjuvant in the process. Despite weight-loss attempts being common practices and Brazil being considered a major consumer of anorectic drugs, population-based studies are still scarce.

Methods Between January and May 2010 we conducted a crosssectional population-based study with household interviews among adults in Pelotas, a medium size city located in southern Brazil, to determine the prevalence of weight-loss attempts in the last year and identify substances most commonly used.

Results Data were collected from 2732 individuals, with a rate loss of $10.3 \%$. Overall prevalence of anti-obesity agents use was $4.8 \%$ (4.0 to 5.7), being higher in women (7.3\%, CI 6.0 to 8.6) and among those of higher schooling $(9.3 \%$, CI 7.0 to 11.7$)$. Drugs mainly used were: sibutramine (45.5\%) amphetamines (31.8\%) and herbal medicines $(25.0 \%)$. Weight-loss formulae containing combinations of drugs, illegal in Brazil, were reported by $6.1 \%$ of users. Only $39.4 \%$ of drugs users referred to add dietary changes and regular exercise, and $47.0 \%$ slimming teas.

Conclusion Despite the regulations, medical prescription was just reported by $49.6 \%$ of the users. Moreover, we found a high prevalence of cardiovascular risk factors among users of sibutramine and amphetamines (hypertension: 30.9\%; Hypercholesterolaemia: 27.7\%; Diabetes: $10.8 \%$; Age > 40 years: $36.6 \%$; Smoking: $10.4 \%$ ) with $5 \%$ of sibutramine users reporting previous myocardial infarction or angina, contra indications for it usage.

\section{P1-335 LONG-TERM CUMULATIVE RISK OF HERPES ZOSTER AMONG IMMUNOCOMPETENT ADULTS 60 YEARS AND OLDER}

doi:10.1136/jech.2011.142976f.27

N Smith, ${ }^{*}$ H F Tseng. Kaiser Permanente Southern California, Pasadena, California, USA

Introduction Herpes zoster (HZ) is a painful disease affecting mostly seniors. The goal of this study is to estimate the long-term cumulative risk of $\mathrm{HZ}$ in a general population 60 years and older.

Methods We conducted a retrospective cohort study of 227277 randomly selected immunocompetent subjects from Kaiser Permanente Southern California members who were never vaccinated with a zoster vaccine, and followed them from 1 January 2007 to 31 December 2009. We estimated the age-specific incidence rates, and used density method to derive the long-term cumulative risk of HZ, assuming the incidence rate is stable over time and no cohort effect. Results The 10-, 20-, and 30-year risks of $\mathrm{HZ}$ are 0.10 (95\% CI 0.09 to -0.11 ), 0.21 ( $95 \%$ CI 0.19 to 0.24 ), and 0.34 ( $95 \%$ CI 0.30 to 0.38 ) for White men, and 0.14 (95\% CI 0.13 to 0.16 ), 0.30 (95\% CI 0.27 to
0.32 ), and 0.42 (95\% CI 0.39 to 0.46 ) for White women, respectively. For Black men, the 10-, 20-, and 30-year risks are 0.08 (95\% CI 0.06 to 0.10 ), 0.17 ( $95 \%$ CI 0.12 to 0.22 ), and 0.23 (95\% CI 0.15 to 0.33 ), and for Black women, they are 0.09 (95\% CI 0.07 to 0.12$), 0.20$ (95\% CI 0.16 to 0.25 ), and 0.30 (95\% CI 0.23 to 0.38 ).

Conclusions As the ever-increasing number of baby boomers is entering the retirement years, the number of elderly population is expected to increase. We estimated that the long term risk of $\mathrm{HZ}$ among people over age of 60 years is higher than previously expected. It may be prudent to suggest prevention measures such as vaccination to reduce the risk of $\mathrm{HZ}$ in this population.

\section{P1-336 VALIDATION OF NHS HOSPITAL ADMISSION RECORDS FOR ISCHAEMIC HEART DISEASE IN THE MILLION WOMEN STUDY}

doi:10.1136/jech.2011.142976f.28

L Wright, ${ }^{*}$ J Green, D Canoy, B Cairns, A Balkwill, V Beral. University of Oxford, Oxford, UK

Introduction The Million Women Study is a large longitudinal UK study of women's health. Between 1996 and 2001, 1.3 million women mostly aged 50-64 years were recruited through NHS breast screening centres in England and Scotland. Their current average age is 68 years. For all participants, the study has linkage to hospital admission records (Hospital Episode Statistics [HES] for England, Scottish Morbidity Records) as one source of study outcomes. Ischaemic heart disease (IHD) is one of the leading causes of disability and death in women in their 60s. However, the accuracy of capturing IHD in women using hospital admission records unclear.

Methods The aim of this study is to validate the occurrence or absence of IHD in HES records in Million Women Study participants using general practice records. A random sample of 1000 women with a hospital record including an IHD diagnosis (ICD I20-I25) and 1000 women with no such admission (controls) were selected. We wrote to each woman's registered general practitioner (GP) asking for confirmation of an IHD diagnosis or not. Each GP was asked to complete a study form, supply relevant hospital documents and return these in a prepaid reply envelope. For each admission, HES data will be compared with GP data.

Results Response rates were $83 \%$ for IHD and $88 \%$ for controls. Results will include analysis of the agreement between general practice medical records and HES admissions data for both cases and controls. The implications of using administrative datasets for epidemiological research outcomes will be discussed.

\section{P1-337 SOCIAL INEQUALITY IN MORTALITY: CAUSALITY OR SELECTION?}

doi:10.1136/jech.2011.142976f.29

${ }^{1} \mathrm{G}$ Søndergaard, ${ }^{*} \mathrm{~L} \mathrm{H}$ Mortensen, ${ }^{2} \mathrm{~A} \mathrm{M}$ N Andersen, ${ }^{1} \mathrm{M}$ Osler. ${ }^{1}$ Research Centre for Prevention and Health, Glostrup, Denmark; ${ }^{2}$ Section for Social Medicine, Department of Public Health, University of Copenhagen, Copenhagen, Denmark

Introduction The aim of this study was to examine to which degree the often demonstrated association between educational status and mortality is confounded by family characteristics, that is environment in childhood and genetic setup. To disentangle an independent causal effect of educational status on mortality from selection mechanisms, we used a discordant sibling design making it possible to control for the shared early life environment and to a lesser degree genetic setup per design.

Methods The study was register-based and the study population consisted of all individuals born in Denmark between 1950 and 1979 